\title{
Similar Algorithms in Different Sensory Systems and Animals
}

\author{
M. KONISHI \\ Division of Biology, California Institute of Technology, Pasadena, California 91125
}

Animals must both recognize and localize the sensory signals essential for their survival and reproduction. Certain cues contained in a signal define its identity and location. Such cues include, for example, the temporal pattern of song for species recognition in crickets and binaural disparities for sound localization in owls. One can predict potential cues and the methods of using them from consideration of the physical attributes of the signal for the task. The discovery of the real cue that is used by the animal, however, requires study of the animal's response to different potential cues.

Once the real cue is identified, the next question is how is it encoded in the language of the nervous system? One can develop an algorithm for the solution of the coding problem. There are, however, many possible ways of solving the same problem. There is, however, no theoretical means of identifying the real algorithm. Analysis of neuronal responses to stimuli and study of the connections between neurons may inform us about the coding scheme because the connections and signals between neurons underlie the coding mechanisms, but what neurons tell depends on what question the investigator asks. As Barlow (1972) pointed out, ". . . neurophysiology and sensation are best linked by looking at the flow of information rather than simpler measures of neuronal activity."

A neurophysiological investigation of how information is transformed from lower- to higher-order stations may be difficult because information processing is highly nonlinear. Going in the opposite direction, the "topdown" approach is easier in some systems because one starts with the knowledge of what is encoded at the top or at a relatively higher station. The criterion for encoding is the stimulus selectivity of single neurons. The flow of information is therefore inferred from the stimulus selectivities recorded in interconnected stations. For example, the neurons of the external nucleus of the inferior colliculus in the barn owl respond selectively to a combination of interaural time and intensity differences. The neuronal selectivities for these binaural cues have been traced from this nucleus back to the first sites where the selectivities emerge and the pathways for the flow of information can be established (Konishi et al. 1988).

The approach that looks for the flow of information has been used only in a few complex neural systems including the visual system of the macaque monkey (for review, see Van Essen 1985; Hubel and Livingstone 1987; Maunsell and Newsome 1987; De Yoe and Van Essen 1988; Livingstone and Hubel 1987, 1988), the auditory system of the barn owl (hereafter referred to as the owl) (for review, see Konishi et al. 1988), and the electrosensory system of the weakly electric fish, Eigenmannia (hereafter referred to as the electric fish) (for review, see Heiligenberg 1986). Also, the topdown approach from the auditory cortex to lower-order nuclei in the mustached bat is rapidly achieving the same level of understanding that has been reached in the owl and electric fish (Suga 1984, 1988; N. Suga et al., this volume). The coding processes and the behavioral significance of neurophysiological and anatomical findings are, however, better understood in the owl and electric fish than in any other vertebrate system. Comparisons of these systems show that different sensory systems and different animals use similar procedures in the processing of biological signals, although their neural implementations may vary. Similar comparisons have been made previously (Ulinski 1984; Carr 1986). This paper discusses some of these procedures for sensory processing and their implications for the theory of neural coding.

\section{Separation of Neural Codes for Different Stimulus Variables}

Sensory systems separate neural codes for different stimulus variables and configurations. Although a single neuron can carry more than one pulse code as in the auditory nerve of the owl, the processing of different stimulus variables eventually requires the separation of relevant codes. Initial separation of codes occurs in sense organs, ganglia, or in the first station of the brain (Ulinski 1984). The owl's auditory system detects interaural time and intensity differences for sound localization (Fig. 1) (Moiseff and Konishi 1981; Moiseff 1989). The owl's auditory nerve carries the codes for the amplitude, phase, and frequency of sound. Amplitude and phase are encoded by the rate and timing of nerve impulses, respectively. The codes for frequencies are separated in the auditory nerve, since different primary auditory fibers respond to different frequencies. On the other hand, the amplitude and phase codes are not separated in the auditory nerve. Separation of these codes occurs in the cochlear nuclei, the first station in the brain. The owl's cochlear nucleus consists of two 


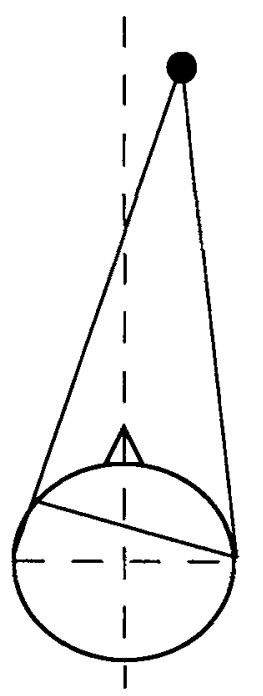

Time difference

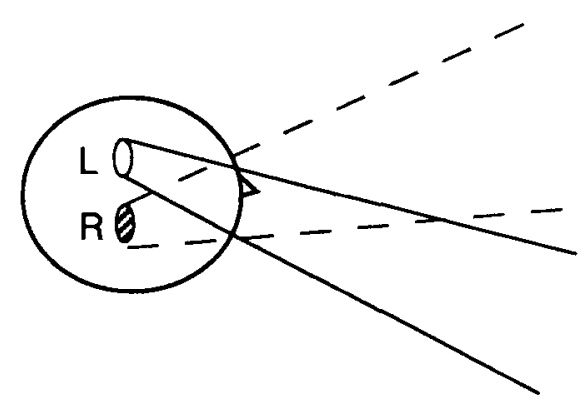

Intensity difference
Figure 1. Binaural disparity cues for sound localization. A difference in the length of sound path between the two ears produces an interaural time difference, which varies as a function of the incidence angle of sound relative to the midsagittal plane of the head. The owl uses this function for localization in azimuth. A difference in the amplitude of sound between the two ears results both from the shadowing effects of the head and from differences in the directionality of the two ears. In the barn owl, the left ear is located higher than the right ear relative to eye level, and the two ears are sensitive in different directions. This asymmetry enables the owl to use interaural intensity differences for localization in the vertical plane. Each two-dimensional locus in space is thus uniquely defined by a combination of interaural time and intensity differences. spatially separate populations of neurons. Both populations receive both the amplitude and phase codes, but one, nucleus angularis, preserves only the amplitude code, and the other, nucleus magnocellularis, largely preserves the phase code (Sullivan and Konishi 1984; Takahashi et al. 1984). The projections from these nuclei to the midbrain station, the inferior colliculus, are separate (Takahashi and Konishi 1989a,b). There are therefore two parallel pathways in the brainstem of the owl.

The electric fish uses sinusoidal electrical signals to orient and navigate in murky water. Fish avoid jamming one another by raising or lowering the frequency of their signals, always changing in the direction that maximizes the frequency difference between the two. A fish detects changes in the amplitude and phase of the beat waveform, resulting from the adding of its own signal and the signal of the neighbor, to determine whether it should lower or raise its frequency to avoid jamming (Fig. 2) (Heiligenberg 1986). Two different classes of electroceptors encode amplitude and phase. Although these receptors are intermixed over the entire body surface, the neurons that receive input from the receptors are separated in different layers of the electrosensory lateral line lobe, the first station of the brain electrosensory pathway. These layers give rise to separate pathways for amplitude and phase, as in the owl.

Similarly, visual neurons sensitive to different stimulus variables are separated in different layers of the retina, the optic tectum, the lateral geniculate body, and the visual cortex (Maturana et al. 1960; Lettvin et al. 1961; for review, see Rodieck 1979). In the macaque monkey's visual system, magnocellular and parvocellular optic ganglion cells have different morphological and physiological properties (Bowling and Michael 1980; Michael 1988). Magnocellular neurons are fast responding and are sensitive to contrast, but they are color blind and poor in spatial resolution, whereas parvocellular neurons are characterized by color selectivity, high spatial resolution, slow response, and low contrast sensitivity. Magnocellular neurons project to the bottom two layers, and parvocellular neurons project to the top four layers of the lateral geniculate body (Kaplan and Shapley 1982; Perry et al. 1984; Michael 1988).

\section{Specialized Neural Circuits for Salient Cues}

The brain contains special circuits to detect salient cues that are not directly encoded in single primary sensory neurons. Examples of these cues include binaural and binocular disparities, orientation, velocity, and echo delays. Although the existence of special circuits for the detection of salient cues would seem obvious, a single neural network that detects more than one such cue cannot be excluded. For example, the oscillator circuits of invertebrates can produce more than one pattern of output by chemical modulation of the synapses and membrane ion channels of selected neurons (Getting 1989; Marder 1989). Whether this sort of modulation regulates the property of sensory detector circuits remains to be investigated.

The existence of special circuits has been proposed in various models of sensory systems, such as circuits for the detection of the direction of stimulus movement in the compound eye of the fly and the rabbit retina, for binocular disparity in the mammalian visual cortex, and for binaural time differences (Jeffress 1948; Reichardt 1961; Barlow et al. 1964; Julesz 1971). The search for the real circuits of this sort has been generally unsuccessful. The circuit for the detection of interaural time differences in the owl's brainstem is a notable exception. Like Jeffress's model, this circuit consists of delay lines and coincidence detectors (Figs. 3 and 4) (Sullivan and Konishi 1986; Carr and Konishi 1988). In the electric fish, circuits for the comparison of the phase angle and amplitude of electrical signals between different body areas have been identified (Maler 1979; Carr et al. 1986a,b; Shumway and Maler 1989). 
A

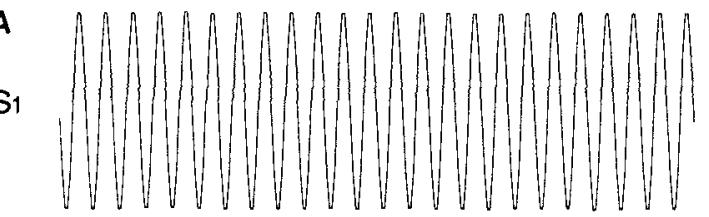

$\mathrm{S} 2$

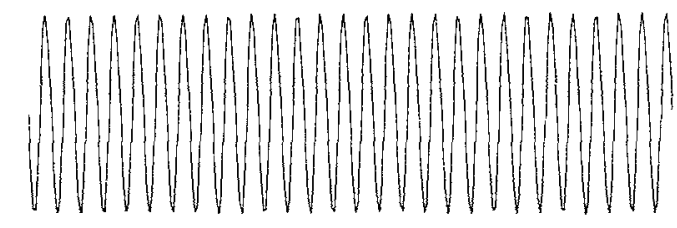

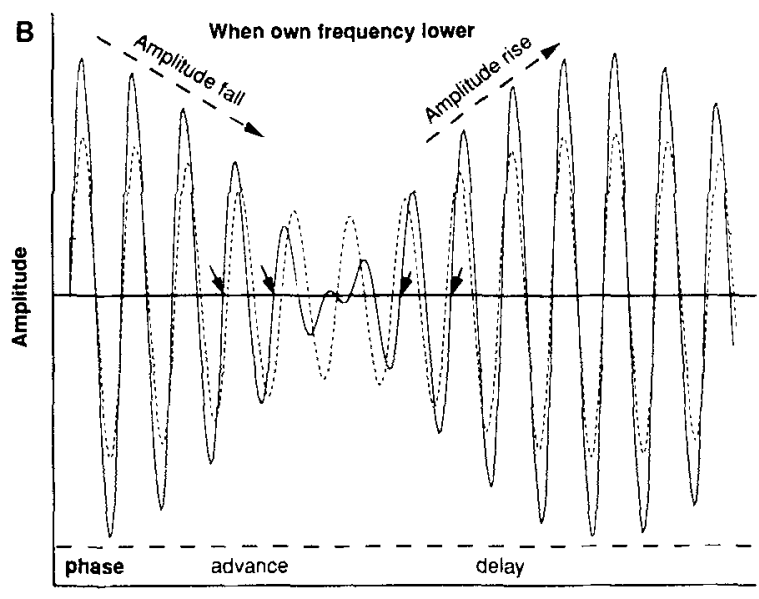

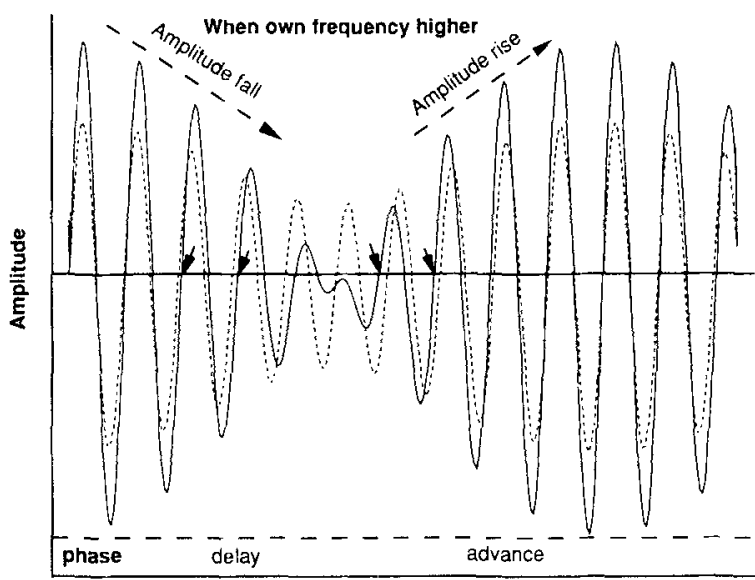

Figure 2. Determination of the sign of frequency differences by electric fish. Eigenmannia. (A) Electrical signals. Eigenmannia generates sinusoidal electrical signals for navigation and orientation. When an individual $\left(S_{1}\right)$ encounters another individual $\left(S_{2}\right)$, they avoid jamming each other by changing the frequency of their signals. The fish uses the beat waveform $\left(S_{1}+S_{z}\right)$ to determine whether its own frequency is higher or lower. $(B)$ The fish uses differences in the phase and amplitude of the beat waveform between different body loci to determine the sign of frequency differences. In this figure, the solid-line and dotted-line waveforms show different degrees of contamination of $S_{1}$ by $S_{2}$. The solid-line waveform is more contaminated and registered at one body locus, and the dotted-line waveform is less contaminated at another locus. The small arrowheads indicate the phase relationships between the two waveforms. The left-slanted arrowheads indicate that the phase of the solid-line waveform is advanced relative to that of the dotted-line waveform. When these phase relationships and the rise and fall of amplitude are combined, the sign of frequency differences can be uniquely determined. Thus, the sequence, a fall in amplitude with a phase advance followed by a rise in amplitude with a phase delay, indicates that the fish's own frequency is lower than that of the other fish.

\section{Transmission of Information by Coded Lines}

Eventual transformation of codes to "coded lines" is a universal operation in all sensory systems. In this scheme, relevant sensory information is transferred from one neuron to the next, not because the recipient neuron sorts out signals encoded in impulses, but simply because the two neurons are connected. The time comparison circuits of owls and electric fish mentioned above receive information about phase from phaselocked spikes. The output of each phase-disparity circuit of the electric fish carries the code for a "phase difference," but this code no longer uses the timing of impulses (cf. Fig. 3). Although the output fibers of the owl's nucleus laminaris carry phase-locked spikes, the phase information so conveyed is not used by the recipient stations. The neurons of these stations lack the morphological specializations necessary for the pre- servation of phase information such as calycine synapses, yet different neurons respond selectively to different ranges of interaural phase disparities. All higherorder neurons that receive input directly or indirectly from the nucleus laminaris are selective for interaural time difference.

New coded lines emerge at all levels. Sound frequency is not encoded in the number of impulses but by coded lines, which ultimately derive their frequency selectivities from the electromechanical properties of the basilar membrane-hair cell complex. Neuronal selectivities for movement, stimulus orientation, and binocular disparity emerge for the first time in the primary visual cortex in the macaque monkey, and color, movement, and orientation-selective neurons are segregated in different layers or areas. These selectivities are line-coded and conveyed by parallel channels to higher-order stations in the extrastriate visual 


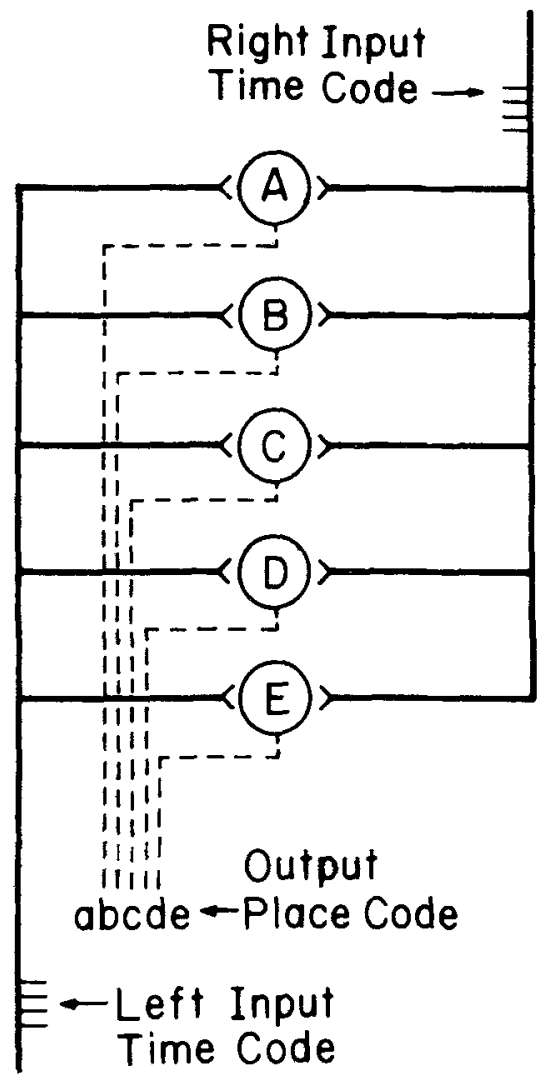

Figure 3. A model of neural circuits for the detection of interaural time differences. It uses the principles of coincidence detection and delay lines. Binaural neurons, A, B, C, $D$, and $E$, fire maximally only when impulses from the two sides arrive simultaneously. Except for $\mathrm{C}$, the paths for impulse transmission to each neuron are different between the two sides. These asymmetries cause interaural differences in the arrival time of impulses. A neuron fires maximally when an imposed interaural time difference compensates for the asymmetry in impulse transmission time. This array of neurons thus encodes systematically different azimuthal locations of sound.

cortex (Hubel and Livingstone 1987; Maunsell and Newsome 1987; De Yoe and Van Essen 1988; Livingstone and Hubel 1988).

The above comparison of the starting points of coded lines shows that code transformation to coded lines occurs as soon as relevant stimulus properties are detected. Just as neural codes for primary stimulus variables are separated, so are those for line-coded cues. Thus, the establishment of coded lines leads to the separation of neural codes for different cues. Linecoding obviates the need to reproduce at different levels of neural systems the same detection mechanisms every time the relevant information is needed.

\section{Convergence of Parallel Pathways}

Parallel pathways for the separate processing of different cues may reunite in higher-order stations. Such convergence serves several different purposes. In the owl's auditory system, interaural time differences are detected in each frequency band by the special circuit mentioned above. Interaural time differences are measured as phase differences in different frequency channels. These channels are separate in all stations below the external nucleus of the inferior colliculus. In these lower stations, neurons respond to more than one interaural time difference because the time differences separated by integer multiples of the stimulus period give rise to the same phase difference. This phenomenon is termed phase ambiguity. The convergence of different frequency channels on single neurons in the external nucleus of the inferior colliculus eliminates phase ambiguous responses in these neurons, thus enabling them to encode the true interaural time difference (Takahashi and Konishi 1986).

Similarly, the electric fish must gather phase and amplitude information from a large area of its body surface to determine the sign of frequency differences. If a fish is experimentally prevented from receiving electrical signals from a large area of its body surface, it cannot discriminate between the signs of frequency differences. Under these conditions, neurons of the prepacemaker nucleus that are sensitive to differences in frequency fail to detect the sign of frequency differences. These uncertainties are partly due to the fact that phase disparities between different body areas vary with the relative orientation of the two fish. The neurons of the prepacemaker nucleus can determine the sign of frequency differences independently of fish orientation because they normally receive inputs from a large part of the body surface (Kawasaki et al. 1988b; Keller 1988; Keller and Heiligenberg 1989). Similarly, the convergence of parallel channels in the visual system appears to underlie the position-independent responses of neurons to stimulus properties, such as orientation (Hubel and Wiesel 1962), and complex stimulus configurations, such as hands and faces (Gross et al. 1972; Perret et al. 1982; Kendrick and Baldwin 1987).

Another role of convergence is the creation of new coordinate systems and stimulus dimensions in sensory perception. The convergence of the intensity and timeprocessing pathways in the owl gives rise to neurons that respond neither to interaural time nor to intensity differences alone but to a combination of the two. These neurons encode auditory space because the combinations of time and intensity differences define the coordinates of auditory space in the owl. In the monkey visual system, the magnocellular and parvocellular pathways appear to converge in several different areas of the cortex (Van Essen 1985). These points of convergence may be responsible for such phenomena as the detection of structure from motion and shape from shading (De Yoe and Van Essen 1988).

\section{Hierarchy, Single Neurons, and Networks}

Sensory systems process stimuli in hierarchically organized neural networks. When a system contains parallel pathways, each pathway may be hierarchically organized. In a hierarchical system, neurons at higher 


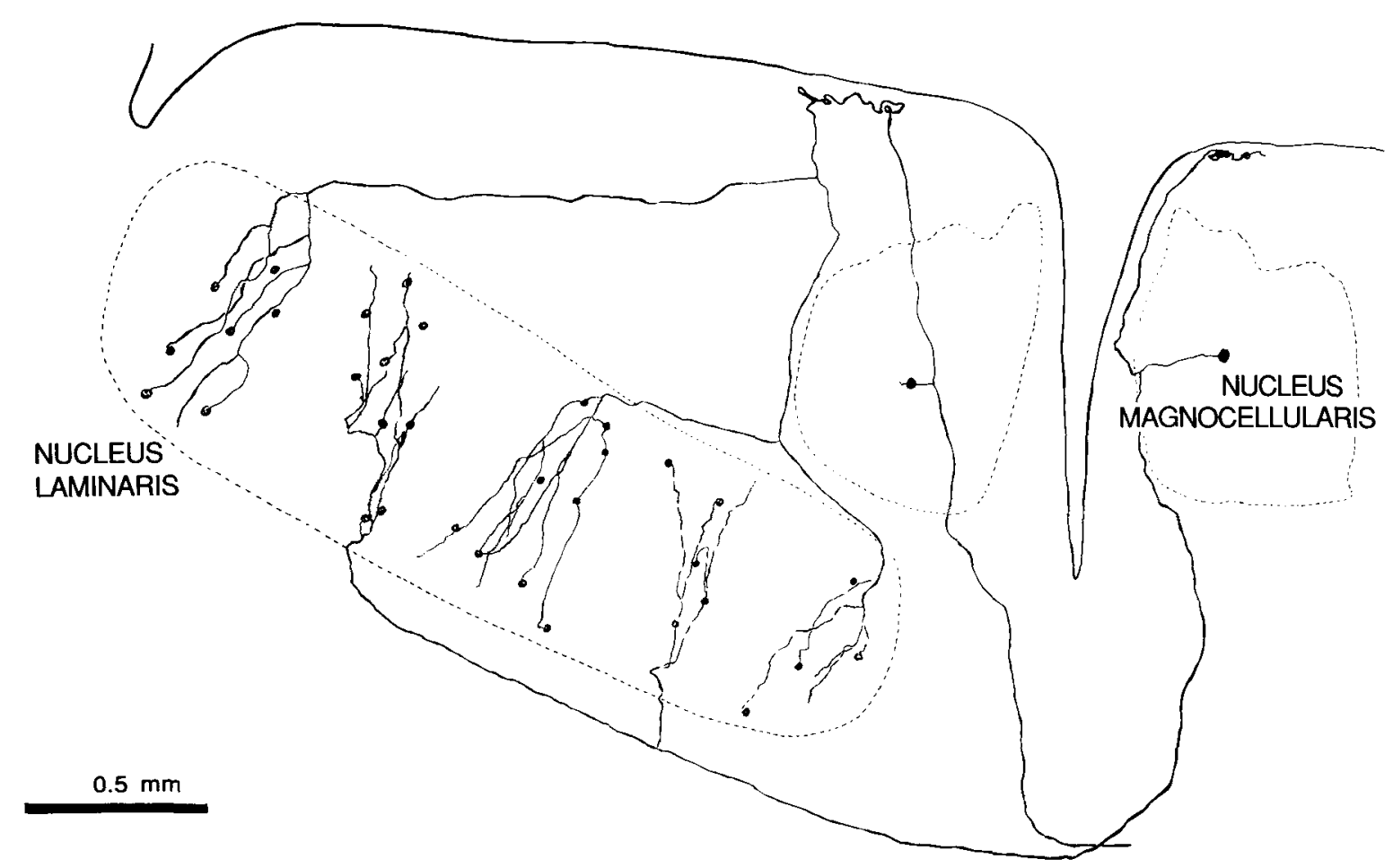

Figure 4. Neural circuits for the detection of interaural time differences. Nucleus magnocellularis is one of the first brain stations in the owl's auditory system. Nucleus laminaris receives inputs from both the ipsilateral and contralateral magnocellular nuclei. The figure shows axon collaterals from single ipsilateral and contralateral neurons projecting into nucleus laminaris, which contain binaural neurons. For the sake of clarity, the ipsilateral and contralateral axons are shown separately, although they interdigitate in reality. These interdigitating axons serve as delay lines, and the laminaris neurons serve as coincidence detectors. Interaural phase differences are computed separately for each frequency band.

stations are selective for more complex combinations and configurations of stimulus variables than those at lower stations. The neuron at the top of the hierarchy represents the results of the sum total of the computations that take place in processing the signal. Sherrington (1941) called such a neuron a "pontifical cell," although he rejected the idea of mind represented by one such cell. The argument against the usefulness of the concept of a pontifical neuron was best expressed by Marr (1982) when he wrote, ". . . Suppose, for example. that one actually found the apocryphal grandmother cell (a cell that fires only when one's grandmother comes into view). Would that really tell us anything much at all? It would tell us that it existed-Gross's hand-detectors tell us almost thatbut not why or even how such a thing may be constructed from the outputs of previously discovered cells. Do the single-unit recordings - the simple and complex cells - tell us much about how to detect edges or why one would want to, except in a rather general way through arguments based on economy and redundancy? If we really knew the answers, for example, we should be able to program them on a computer. But finding a hand-detector certainly did not allow us to program one."

One of the problems in the study of these "objectspecific" neurons is to identify the salient cues that the neurons and animal detect for the recognition of the object. Once the cues are defined, the origin and trans- mission of neuronal selectivities for them can be studied. For further discussion of object-specific neurons, there are two points that need clarification. One is the expression, single neurons; a single cell may be selective for a complex stimulus, but it does not perform all the computations necessary for the recognition of the stimulus. A single neuron in a network is nothing but a nodal point, although the point may be a site of complex integrative processes. Tapping of such a point can reveal the results of some of the computations carried out by the network. When a network is hierarchically organized, the neuron at the top of the hierarchy will represent the results of all computations by the network. The second point concerns the idea that there ought to be only one cell at the top of the hierarchy. Because there is no theoretical reason to reject such an idea, one must rely on the results of observational and experimental studies.

In both the owl and electric fish, we know the algorithms for the genesis of the stimulus selectivity of neurons at the top of the hierarchically organized networks. In other words, we know the connections and processes underlying the stimulus selectivity of these object-specific neurons. Figure 5 summarizes the algorithm for sound localization by the owl and that for jamming avoidance response by the electric fish. In this figure, the hierarchy of processing can be compared with that of networks. Thus, in these systems, the neurons at the top of the hierarchy do represent the 

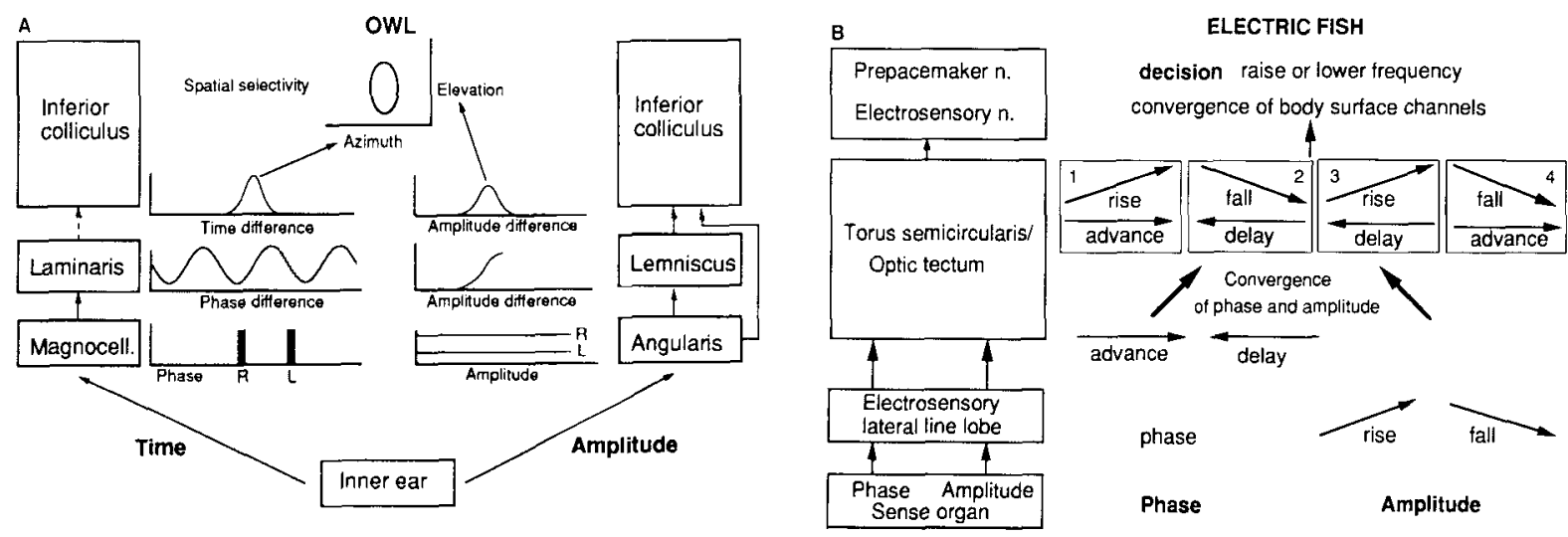

Figure 5. Hierarchical organization. In both the owl's auditory system $(A)$ and the electric fish's electrosensory system $(B)$, biologically relevant signals are processed in hierarchically organized neural networks. Primary stimulus variables, such as the phase and amplitude of sinusoidal signals, are encoded in the sense organ. The neural codes for these stimulus variables are routed to different parallel pathways in the first brain station. The stimulus configuration for the detection of relevant signals are detected by special neural circuits in higher-order stations. The parallel pathways that process different cues for the detection of the same signal converge in higher-order stations, where neuronal selectivity for a combination of the different cues emerges. In general, neuronal stimulus selectivity becomes narrower and less ambiguous as one ascends the hierarchy. The stimulus selectivity of the neurons at the top of the hierarchy is the result of all the computations that are carried out by the networks. The stimulus selectivity of these neurons is very much like that of the whole animal. In $A$, the ordinate of all graphs shows the number of impulses. The bar histogram shows the sensitivity of neurons to stimulus phase. Phase information is conveyed to both cochlear nuclei, but only the nucleus magnocellularis retains the phase code. The two magnocellular nuclei send the right (R) and left (L) phase values in each frequency band to nucleus laminaris, which detects and encodes phase differences. The sinusoidal curve next to nucleus laminaris shows neuronal responses to interaural time differences that give rise to the same phase difference. The cochlear nucleus angularis abandons the phase code but retains the amplitude code; the line graph shows how a right and left intensity difference is encoded in the discharge rate of angularis neurons. This information is sent to one of the lemniscal nuclei and the inferior colliculus. Partial selectivity to interaural intensity differences emerges in the lemniscal nucleus (Manley et al. 1988). Convergence of the time and intensity pathways in the inferior colliculus creates neurons selective for a combination of intensity and time disparities. These neurons have spatial receptive fields in which the azimuthal and elevational position and dimension are determined, respectively, by the neurons' selectivity to interaural time and intensity differences. In $B$, the arrows pointing to the right and left indicate neurons sensitive to phase advance and delay, respectively. These neurons occur in lamina 6 of the torus semicircularis. Numbers 1,2,3, and 4 indicate four different neuron types (in deeper layers of the torus semicircularis) that are selective for four different combinations of phase and amplitude combinations mentioned in Fig. $2 \mathrm{~B}$. The prepacemaker nucleus contains neurons that discriminate the sign of frequency differences.

final results of the computations that occur in all of the lower-order networks (Konishi et al. 1988; Rose et al. 1988). In both the owl and the electric fish, experimental disruptions of any of these computational processes have profound effects on the selectivity of the neurons at the top of the hierarchy (Takahashi et al. 1984; Heiligenberg 1986).

The hierarchies shown in Figure 5 appear simple without feedback loops, although such connections are known in the electric fish (Bastian 1986; Carr and Maler 1986; Bastian and Bratton 1990; Bratton and Bastian 1990). Hierarchical organizations are, however, evident in even more complex systems such as the macaque visual system in which reciprocal connections occur between almost all successive levels above the lateral geniculate nucleus. The anatomical hierarchy in this case is recognized by the feedforward and feedback connections that can be recognized in the laminar organization of the cortex (Maunsell and Van Essen 1983). The hierarchy of processing in the monkey visual system can be inferred from the distribution of different stimulus selectivities in interconnected stations. For example, the lateral geniculate nucleus is anatomically one level below the primary visual cortex. The same rank order applies to these stations in terms of processing because neuronal selectivity for orientation and the direction of movement is not present in the lateral geniculate but emerges for the first time in the primary visual cortex. Similarly, neuronal selectivity for stimulus velocity emerges in the middle temporal (MT) area which is anatomically at least two levels away from the primary visual cortex (Hubel and Livingstone 1987; Maunsell and Newsome 1987; De Yoe and Van Essen, 1988; Livingstone and Hubel 1988), although the contributions of inputs other than the striate cortex and V2 to the stimulus selectivities of MT neurons make a simple serial hierarchy unlikely (Rodman et al. 1989).

\section{Brain Maps and Coding}

The owl's space-specific neurons are arranged according to the location of their spatial receptive fields, i.e., they form a map of auditory space (Knudsen and Konishi 1978a). A similar kind of map is found in the mustached bat; this species presumably measures the distance of a target by detecting the time delay between the emitted signal and the echo from the object. In the auditory cortex of this species, echo delays are mapped, 
i.e., neurons tuned to different echo delays are systematically arranged (Suga and O'Neill 1979).

Neither the map of auditory space nor the map of echo delays is due to a topographical projection of the inner ear, which maps frequency instead of space or time. The maps of auditory space and echo-delays are "centrally synthesized" in contrast with maps that copy the topographical arrangement of sensory cells, such as the tonotopic, retinotopic, and somatotopic maps (Konishi 1986; Knudsen et al. 1987). In a centrally synthesized map, the mapped variable is computed by a special network in a lower-order station or within the station containing the map, and different values of the variable are systematically represented by an orderly array of neurons.

Neuronal maps present an interesting problem for the study of the coding mechanisms. All maps contain place-codes, meaning that spike number, intervals, and other parametric or stochastic attributes do not uniquely distinguish one member neuron from another. Take, for example, two space-specific neurons at some distance apart in the map; they are tuned to different combinations of interaural time and intensity differences, yet they may fire the same number of spikes with the same temporal pattern. The map sites where firing occurs is thus the only code for the location of the sound source. A neuronal map is, however, not just a two-dimensional matrix of independent variables, but an array with connections between the member neurons. Neurophysiological evidence suggests that the neighboring space-specific neurons of the owl interact to produce a receptive field organization consisting of an excitatory center and an inhibitory surround (Knudsen and Konishi 1978b). Such an interaction may be mediated by the presumed "horizontal connections" between space-specific neurons.

Although brain maps would seem most attractive for the study of distributed coding mechanisms, there is no reason to believe that such mechanisms involve the whole map. What is the minimum map area over which integration must take place for relevant sensations to occur? In addressing this question, one can learn much from behavioral studies. For example, the preattentive discrimination of visual textures uses local features rather than the statistical attributes of the textures encompassing the whole visual field (Julesz 1981).

\section{Object-specific Neurons and Perception}

The space-specific neurons of the owl and the prepacemaker neurons of the electric fish are selective for the same salient cues that cause the whole animal to experience the percept of the relevant signal or object. The behavioral role of object-specific neurons either can be inferred from comparison between behavioral and neuronal findings or can be studied directly by ablation or stimulation of the neurons. The stimulus requirements and response properties of these neurons may match qualitatively or even quantitatively with the behavioral responses of the animal. This match has been particularly well established in the electric fish. As mentioned above, just as the fish can behaviorally determine the sign of frequency differences independently of the orientation of the electrical field, so can the neurons of the prepacemaker nucleus. This ability contrasts with the inability of neurons in lower-order stations to distinguish unambiguously one sign from the other. The electric fish is extremely sensitive to differences in the phase angles of the electrical signals between different loci on the body surface. Evidence shows that the fish can behaviorally detect a time difference as small as $1 \mu \mathrm{sec}$. The prepacemaker neurons are equally sensitive to such small time differences under the same experimental conditions (Rose and Heiligenberg 1985; Kawasaki et al. 1988a).

In the owl, space-specific neurons respond only to sound coming from a restricted area in space because they are tuned to the combination of interaural time and intensity differences that results when the sound source is located in the restricted area. Similarly, a combination of interaural time and intensity differences causes the owl to turn its head in the direction predicted from the binaural disparities. The owl hears two signals, one in each ear, yet it "perceives" a single phantom source, when the two signals are identical except for disparities in time and intensity between the ears. The same phenomenon in man is known as binaural fusion. The connections and signals of the network that the space-specific neurons represent underlie the perception of location, and the activity of these neurons presumably creates the percepts of spatial loci.

Although comparisons of behavior and neuronal responses provide indirect evidence for the perceptual role of object-specific neurons, direct evidence is difficult to obtain. Lesions and stimulation are usually regarded as the ultimate means of testing the behavioral function of neurons. For example, the middle temporal area of the macaque monkey contains many neurons selective for stimulus velocity. A partial lesion of this area temporarily abolishes the learned response of the eye to pursue a moving stimulus and raises the threshold of detecting coherent movement in a random dot display. The recovery from the effects of lesions remains unexplained (Newsome et al. 1985; Newsome and Pare 1988).

\section{Object-specific Neurons in Sensorimotor Transformation}

The formation of a percept may cause effector or motor responses, as in the raising or lowering of signal frequency in the electric fish and in the sound-induced head-turning response of the owl. The owl's auditory system transforms binaural disparities into headcentered spatial coordinates. These space-specific neurons project to the optic tectum where they form a joint auditory-visual map of space (Knudsen 1982). In addition, the optic lobe appears to contain maps of head movement vector and speed (Du Lac and Knud- 
sen 1990). How the bimodal map projects to the motor map is not known.

The prepacemaker nucleus of the electric fish is particularly interesting for the discussion of central sensorimotor links (Heiligenberg 1990; Keller et al. 1990). The nucleus contains two classes of neurons: one concerned with the jamming avoidance response and the other with aggression and courtship behavior. The fish modulates its electrical signal to produce "chirps" during courtship. Intracellular stimulation of a single neuron elicits chirps in the fish. Although these neurons fire during spontaneous chirps, an individual neuron may or may not fire. There are about $100-200$ such cells in each side of the body, and different subsets of cells might control different chirp patterns. These examples from the owl and electric fish illustrate how objectspecific neurons can convey the output of a hierarchically organized sensory system to a specific motor system, which itself is hierarchically organized. In other systems, higher-order interneurons link hierarchically organized sensory and motor systems. Electrical stimulation of a single interneuron in the ventral nerve cord of a crayfish elicits a particular posture or movement (Wiersma and Ikeda 1964). According to recent studies of command neurons in the behaving animal, a group of command fibers contribute to the control of several different motor output patterns, but the amount and nature of the contributions by different fibers vary in different patterns (Hensler 1988; Larimer 1988). Similar principles appear to be used in the cortical control of the arms, hands, and fingers in the macaque monkey (Lemon 1988).

\section{CONCLUDING REMARKS}

Contrary to the growing skepticism that the method of single-unit recording is inadequate for the study of complex networks, the proper use of single-unit neurophysiology is in the analysis of network property and function. The two main examples used here show that the single-unit method is a powerful tool for the study of complex networks when certain conditions are met. These prerequisites include knowledge of the salient cues used by the animal, the possibility of manipulating and using the same cues in both behavioral and neurophysiological experiments, and information about the connections between different stations of the neural system in question. The owl and electric fish systems satisfy all of the conditions. The stimulus selectivity of single neurons provides direct access to the coding mechanisms of these systems. Comparisons of the two systems show similar steps and procedures in the genesis of the neural codes for biologically relevant signals. These similarities are not just due to the common evolutionary source from which the auditory and electrosensory systems descended. More complex systems such as the visual system of the macaque monkey appear to use the same basic steps and procedures. These similarities therefore suggest the existence of rules in neural coding that transcend different neural systems, although the neural implementation of the rules may differ between systems.

Finally, one of the challenges for future research is how to explain dynamic changes in perception such as shifts in attention. In the electric fish, the electrosensory lateral line lobe, the first station in the brain, is controlled by feedback from one of the higher centers, both directly and indirectly via the cerebellum. Preliminary evidence suggests that the indirect feedback pathway may control the gain of all pyramidal neurons, and the direct one may control the gain of local populations of somatotopically arranged pyramidal neurons (Bastian 1986; Bastian and Bratton 1990; Bratton and Bastian 1990). As Heiligenberg (1990) pointed out, local control may help to concentrate attention to the input from a particular part of the body surface. In the case of the owl, it refuses to localize a signal if its head-turning response is repeatedly unrewarded. Similarly, the owl learns to discriminate between rewarding and unrewarding targets presented either simultaneously or separately (Quine and Konishi 1974; Kenuk and Konishi 1975). The site and mechanisms of such decision making and their relationships to the hierarchical system described above remain to be established.

\section{ACKNOWLEDGMENTS}

I thank Allison Doupe and Caroly Shumway for reading the manuscript.

\section{REFERENCES}

Barlow, H.B. 1972. Single units and sensation: A neuron doctrine for perceptual psychology? Perception 1: 371.

Barlow, H.B., R.M. Hill, and W.R. Levick. 1964. Retinal ganglion cells responding selectively to direction and speed of image motion in the rabbit. J. Physiol. 173: 377 .

Bastian, J. 1986. Gain control in the electrosensory system: A role for the descending projections to the electrosensory lateral line lobe. J. Comp. Physiol. A 158: 505.

Bastian, J. and B. Bratton. 1990. Descending control of electroreception. I. Properties of nucleus praeeminentialis neurons projecting indirectly to the electrosensory lateral line lobe. J. Neurosci. 10: 1226.

Bowling, D.B. and C.R. Michael. 1980. Projections of single physiologically characterized optic tract fibres in the cat. Nature 286: 899.

Bratton, B. and J. Bastian. 1990. Descending control of electroreception. II. Properties of nucleus praeeminentialis neurons projecting directly to the electrosensory lateral line lobe. J. Neurosci. 10: 1241.

Carr, C.E. 1986. Time coding in electric fish and barn owls. Brain Behav. Evol. 28: 122.

Carr, C.E. and M. Konishi. 1988. Axonal delay lines for time measurement in the owl's brainstem. Proc. Natl. Acad. Sci. 85: 8311.

Carr, C.E. and L. Maler. 1986. Electroreception in gymnotiform fish. Central anatomy and physiology. In Elec troreception (ed. T.H. Bullock and W. Heiligenberg), p. 319. Wiley, New York.

Carr, C.E., W. Heiligenberg, and G.J. Rose. 1986a. A timecomparison circuit in the electric fish midbrain. 1 . Behavior and physiology. J. Neurosci. 6: 107.

Carr, C.E., L. Maler, and B. Taylor. 1986b. A timecomparison circuit in the electric fish midbrain. II. Functional morphology. J. Neurosci. 6: 1372. 
De Yoe, E.A. and D.C. Van Essen. 1988. Concurrent processing streams in monkey visual cortex. Trends Neurosci. 11: 219 .

Du Lac, S. and E.I. Knudsen. 1990. Neural maps of head movement vector and speed in the optic tectum of the barn owl. J. Neurophysiol. 63: 131.

Getting, P.A. 1989. Emerging principles governing the operation of neural networks. Annu. Rev. Neurosci. 12: 185.

Gross, C.G., C.E. Rocha-Miranda, and D.B. Bender. 1972. Visual properties of neurons in inferotemporal cortex of the macaque. J. Neurophysiol. 35:96.

Heiligenberg, W. 1986. Jamming avoidance responses. In Electroreception (ed. T.H. Bullock and W. Heiligenberg). p. 613. Wiley, New York.

- 1990 . The neural basis of behavior. A neuroethological view. Annu. Rev. Neurosci. 11: (in press).

Hensler, K. 1988. Intersegmental interneurons involved in the control of head movements in crickets. J. Comp. Physiol. 162: 111 .

Hubel, D.H. and M.S. Livingstone. 1987. Segregation of form, color and stereopsis in primate area $18 . J$. Neurosci. 7: 3378 .

Hubel, H.D. and T.N. Wiesel. 1962. Receptive fields, binocular interaction and functional architecture in the cat's visual cortex. J. Physiol. 160: 106.

Jeffress, L.A. 1948. A place theory of sound localization. $J$. Comp. Physiol. Psychol. 41: 35.

Julesz, B. 1971. Foundations of cyclopean perception. University of Chicago Press, Illinois.

1981. Textons, the elements of texture perception, and their interactions. Nature 290: 91.

Kaplan, E. and R.M. Shapley. 1982. X and Y cells in the lateral geniculate nucleus of macaque monkeys. J. Physiol. 330: 125 .

Kawasaki, M., G.J. Rose, and W. Heiligenberg. 1988a. Temporal hyperacuity in single neurons of electric fish. Nature 336: 173 .

Kawasaki, M., L. Maler, G.J. Rose, and W. Heiligenberg. $1988 \mathrm{~b}$. Anatomical and functional organization of the prepacemaker nucleus in gymnotiform electric fish: The accommodation of two behaviors in one nucleus. J. Comp. Neurol. 276: 113 .

Keller, C.H. 1988. Stimulus discrimination in the diencephalon of Eigenmannia: The emergence and sharpening of a sensory filter. J. Comp. Physiol. 162: 747.

Keller, C.H. and W. Heiligenberg. 1989. From distributed sensory processing to discrete motor representations in the diencephalone of the electric fish, Eigenmannia. J. Comp. Physiol. A 164: 565.

Keller, C.H., L. Maler, and W. Heiligenberg. 1990. Structural and functional organization of a diencephalic sensorymotor interface in the gymnotiform fish, Eigenmannia. $J$. Comp. Neurol. 293: 347 .

Kendrick, K.M. and B.A. Baldwin. 1987. Cells in temporal cortex of conscious sheep can respond preferentially to the sight of faces. Science 236: 448.

Kenuk, S.A. and M. Konishi. 1975. Discrimination of noise spectra by memory in the barn owl. J. Comp. Physiol. 97: 55 .

Konishi, M. 1986. Centrally synthesized maps of sensory space. Trends Neurosci. 9: 163

Konishi, M., T.T. Takahashi, H. Wagner, W.E. Sullivan, and C.E. Carr. 1988. Neurophysiological and anatomical substrates of sound localization in the owl. In Auditory function (ed. G.M. Edelman et al.), p. 721. Wiley, New York.

Knudsen, E.I. 1982. Auditory and visual maps of space in the optic tectum of the owl. J. Neurosci. 2: 1177.

Knudsen, E.I. and M. Konishi. 1978a. A neural map of auditory space in the owl. Science 200: 795 .

- $1978 \mathrm{~b}$. Center-surround organization of auditory receptive fields. Science 202: 778 .

Knudsen, E.I., S. du Lac, and S.D. Esterly. 1987. Computational maps in the brain. Annu. Rev. Neurosci. 10: 41
Larimer, J.L.. 1988. The command hypothesis: A new view using an old example. Trends Neurosci. 11: 506.

Lemon, R. 1988. The output map of the primate motor cortex. Trends Neurosci. 11: 501.

Lettvin, J.L., H.R. Maturana, W.H. Pitt, and W.S. McCulloch. 1961. Two remarks on the visual system of the frog. In Sensory communication (ed. W.A. Rosenblith). M.I.T. Press, Cambridge, Massachusetts.

Livingstone, M.S. and D.H. Hubel 1987. Psychophysical evidence for separate channels for the perception of form, color, movement, and depth. J. Neurosci. 7: 3416 .

- 1988. Segregation of form, color, movement, and depth: Anatomy, physiology, and perception. Science 240: 740

Maler, L. 1979. The posterior lateral line lobe of certain gymnotoid fish: Quantitative light microscopy. J. Comp. Neurol. 183: 323.

Manley, G.A., C. Koeppl, and M. Konishi. 1988. A neural map of interaural intensity difference in the brainstem of the barn owl. J. Neurosci. 8: 2665 .

Marder, E. 1989. Chemical modulation of an oscillatory neural circuits. In Neuronal and cellular oscillators (ed. J.W. Jacklet), p. 317. Marcel Dekker, New York.

Marr, D. 1982. Vision. Freeman, San Francisco.

Maturana, H.R., J.L. Lettvin, W.H. Pitt. and W.S. McCulloch. 1960. Physiology and anatomy of vision in the frog. $J$. Gen. Physiol. (suppl.) 43: 129.

Maunsell, J.H.R. and W.T. Newsome. 1987. Visual processing in monkey extrastriate cortex. Annu. Rev. Neurosci. 10: 363 .

Maunsell, J.H.R. and C.D. Van Essen. 1983. The connections of the middle temporal visual area (MT) and their relationship to a cortical hierarchy in the macaque monkey. $J$. Neurosci. 3: 2526.

Michael, C.R. 1988. Retinal afferent arborization patterns, dendritic field orientations, and the segregation of function in the lateral geniculate nucleus of the monkey. Proc. Natl. Acad. Sci. 85: 4914.

Moiseff, A. 1989. Bi-coordinate sound localization by the barn owl. J. Comp. Physiol. 164: 637.

Moiseff, A. and M. Konishi. 1981. Neuronal and behavioral sensitivity to binaural time difference in the owl. $J$. Neurosci. 1: 40 .

Newsome, W.T. and E.B. Pare. 1988. A selective impairment of motion perception following lesions of the middle temporal visual area (MT). J. Neurosci. 8: 2201.

Newsome, W.T., R.H. Wurtz, M.R. Dursteler, and A. Mikami. 1985. Deficits in visual motion processing following ibotenic acid lesions of the middle temporal visual area of the macaque monkey. J. Neurosci. 5: 825.

Perret, D.I., E.T. Rolls, and W. Caan. 1982. Visual neurons responsive to faces in the monkey temporal cortex. Exp. Brain Res. 42: 319 .

Perry, V.H., R. Oehler, and A. Cowey. 1984. Retinal ganglion cells that project to the dorsal lateral geniculate nucleus in the macaque monkey. Neuroscience 12: 1101.

Quine, D.B. and M. Konishi. 1974. Absolute frequency discrimination in the barn owl. J. Comp. Physiol. 93: 347.

Reichardt, W. 1961. Autocorrelation: A principle for the evaluation of sensory information by the central nervous system. In Sensory communication (ed. W.A. Rosenblith), p. 303. Wiley, New York.

Rodieck, R.W. 1979. Visual pathways. Annu. Rev. Neurosci. 2: 193.

Rodman, H.R., C.G. Gross, and T.D. Albright. 1989. Afferent basis of visual response properties in area MT of the macaque. I. Effects of striate removal. J. Neurosci. 9: 2033 .

Rose, G. and W. Heiligenberg. 1985. Temporal hyperacuity in the electric sense of fish. Nature 318: 178.

Rose, G.J., M. Kawasaki, and W. Heiligenberg. 1988. "Recognition units" at the top of a neuronal hierarchy? Prepacemaker neurons in Eigenmannia code the sign of fre- 
quency differences unambiguously. J. Comp. Neurol. 162: 759 .

Sherrington, C.S. 1941. Man on his nature. Cambridge University Press, Cambridge, England.

Shumway, C.A. and L. Maler. 1989. GABAnergic inhibition shapes temporal and spatial response properties of pyramidal cells in the electrolateral line lobe of gymnotoid fish. $J$. Comp. Physiol. 164: 391.

Suga, N. 1984. The extent to which biosonar information is represented in the bat auditory cortex. In Dynamic aspects of neocortical function (ed. G.M. Edelman et al.), p. 315. Wiley, New York.

_. 1988. Auditory neuroethology and speech processing: Complex-sound processing by combination-sensitive neurons. In Auditory function (ed. G.M. Edelman et al.), p. 679 . Wiley, New York.

Suga, N. and W.E. O'Neill. 1979. Neural axis representing target range in the auditory cortex of the mustache bat. Science 206: 351.

Sullivan, W.E. and M. Konishi. 1984. Segregation of stimulus phase and intensity in the cochlear nuclei of the barn owl. J. Neurosci. 4: 1787.
1986. Neural map of interaural phase difference in the owl's brainstem. Proc. Natl. Acad. Sci. 83: 8400.

Takahashi, T.T. and M. Konishi. 1986. Selectivity for interaural time difference in the owl's midbrain. $J$. Neurosci. 6: 3413 .

- 1988a. Projections of the cochlear nuclei and nucleus laminaris to the inferior colliculus of the barn owl. $J$. Comp. Neurol. 274: 190.

. 1988b. Projections of nucleus angularis and nucleus laminaris to the lateral lemniscal nuclear complex of the barn owl. J. Comp. Neurol. 274: 212.

Takahashi, T., A. Moiseff, and M. Konishi. 1984. Time and intensity cues are processed independently in the auditory system of the owl. J. Neurosci. 4: 1781 .

Ulinski, P.S. 1984. Design features in vertebrate sensory systems. Am. Zool. 24: 717 .

Van Essen, D.C. 1985. Functional organization of primate visual cortex. In Cerebral cortex (ed. A. Peters and E.G. Jones), vol. 3, p. 259. Plenum Press, New York.

Wiersma, C.A.G. and K. Ikeda. 1964. Interneurons commanding movements in the crayfish Procambarus clarki (Girard). Comp. Biochem. Physiol. 12: 509. 


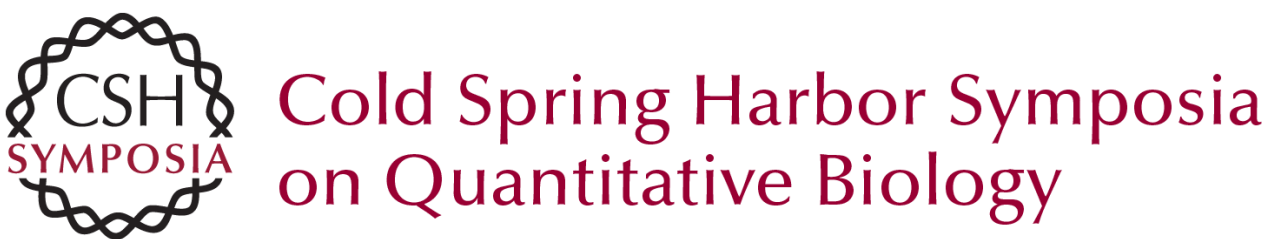

\section{Similar Algorithms in Different Sensory Systems and Animals}

M. Konishi

Cold Spring Harb Symp Quant Biol 1990 55: 575-584

Access the most recent version at doi:10.1101/SQB.1990.055.01.055

References This article cites 64 articles, 27 of which can be accessed free at:

http://symposium.cshlp.org/content/55/575.refs.html

Email alerting Receive free email alerts when new articles cite this article service sign up in the box at the top right corner of the article or click here

To subscribe to Cold Spring Harbor Symposia on Quantitative Biology go to: http://symposium.cshlp.org/subscriptions

Copyright (C) 1990 Cold Spring Harbor Laboratory Press 